\title{
Dossier
}

El trabajo de cuidar y el derecho al cuidado

\section{¿Círculos concéntricos \\ de la política social?}

Laura C. Pautassi

Instituto de Investigaciones Jurídicas y Sociales "A. Gioja"

Facultad de Derecho (UBA)

Abogada (UNC)

Doctora en Ciencias Sociales (UBA)

Investigadora Independiente (CONICET)

Directora del Proyecto Interdisciplinario UBACYT

"Derechos sociales, provisión de bienestar y marginaciones sociales en Argentina"

Integrante del Equipo Latinoamericano de Justicia y Género (ELA)

E-mail: laurapautassi@derecho.uba.ar - www.dspp.com.ar 
No es novedad constatar que la mayor incorporación de la mujer en el mercado de trabajo remunerado ha sido uno de los grandes cambios de escenario económico, social y político en América Latina desde fines del siglo pasado. Tampoco resulta novedoso que este proceso se produce de manera silenciosa, pero sostenida, especialmente cuando las demandas de cuidado han seguido recayendo en forma prácticamente exclusiva en las mujeres. Esto es, la gestión de un deseado "equilibrio" entre demandas del trabajo productivo y las de cuidado siguen ligadas a las mujeres, a pesar de una acción comunicativa que recupera y refuerza el mito de una organización económica y social neutral al género perpetuada tanto desde los discursos oficiales como desde la dinámica social.

La importante evidencia empírica de que disponemos, demuestra cómo las regulaciones laborales presentan un claro sesgo de género, donde el tratamiento de estas medidas es sólo para "mujeres" presuponiendo las leyes laborales que los hijos e hijas son responsabilidad exclusiva de ellas y excluyendo por completo la consideración de otros sujetos que demandan cuidado como las personas adultas mayores, personas con discapacidad o enfermos (Pautassi et al, 2004; PNUD, 2009; Rico y Pautassi, 2001); lo cual confirma una vez más que no existe la argumentada neutralidad. Y a ello se suma la baja conciencia de los empleadores de cumplir con las obligaciones vinculadas a las responsabilidades trabajo/familia, el frecuente $-y$ a su vez falaz- argumento del mayor costo laboral que representaría la maternidad, que ha quedado claramente demostrado no es más que un mito (Abramo y Todaro, 1999; Rodríguez Enríquez, 2009), la baja capacidad de fiscalización del Estado, pero principalmente la falta de consideración de que la maternidad, pero también el cuidado de enfermos o personas adultas mayores, no son cuestiones individuales sino funciones sociales y que interpelan la división sexual del trabajo en la sociedad (Folbre, 2000; Torns Martin, 2005; Tronto, 2006).

Significa que los arreglos que cada familia, y dentro de las familias las mujeres, han encontrado para resolver el cuidado de los familiares 
dependientes -que incluye a sus propios cónyuges en tanto ellos no asumen las tareas necesarias de la reproducción social y tampoco las tareas específicas de cuidado- va a depender de la disponibilidad de ingresos y recursos, promoviendo una nueva desigualdad y un "círculo vicioso": quienes tienen más recursos pueden cuidar mejor y cuidarse y protegerse más, en desmedro, claro está, de las mujeres de menores recursos que además tienen en general más personas bajo su cuidado y menos tiempo para el autocuidado. Es la imagen que se trasluce hace un tiempo: "se cuida como se puede, se es cuidado también como y cuando se puede".

Concordantemente, resulta central considerar que el cuidado es un derecho universal que incluye a todos y a todas, en su potestad de reclamar el derecho a ser cuidado, a cuidar y a cuidarse (autocuidado) (Pautassi, 2007). Significa que no se trata sólo de impulsar acciones que aumenten la oferta de servicios reproductivos, imprescindibles sin lugar a dudas y no sólo en los núcleos urbanos, sino que transversalmente se aborde las responsabilidades de cuidado que le competen a cada miembro de la sociedad, desde el Estado, los empleadores, las organizaciones sociales y comunitarias (OSyC) y los miembros de la pareja, pero también la potestad que tienen de reclamar ser cuidados o las condiciones para ejercer el autocuidado, desvinculando de este modo al cuidado de las relaciones laborales asalariadas y ubicarlo en titularidad de cada persona.

Sin embargo, y tal como desarrollo a continuación, desde las políticas públicas no se discuten arreglos institucionales en esta dirección, ya que en general hay una ausencia de la mirada de las instituciones de política social sobre la promoción de cambios en la organización de la vida cotidiana, como por ejemplo adaptar servicios y transporte públicos, horarios escolares, sistema de salud, que permitan articular de mejor manera las demandas laborales con las de cuidado, pero que no se limiten a esta esfera sino que deslinden la responsabilidad exclusiva del cuidado de las mujeres.

A lo largo del artículo busco demostrar cómo la discriminación estructural en la participación social, política y económica de las mujeres ha configurado una entramado en donde las mujeres tienen 
escasas posibilidades de poder desarrollarse de manera autónoma y donde, a su vez, las dinámicas actuales imperantes poco avanzan en diseñar arreglos institucionales que permitan erradicar la desigualdad estructural. Para ello, y en base al análisis del caso argentino post convertibilidad, busco identificar los principales aspectos que consolidan las asimetrías en el ejercicio de una vida autónoma para las mujeres, a partir de una lectura de la dinámica de comportamiento del mercado de trabajo remunerado y de las formas en que se cuida a las personas, buscando identificar los temas claves de una agenda social, política y económica superadora de las discriminaciones existentes.

\section{Entornos inequitativos: el mercado de trabajo}

Considerando que la división sexual del trabajo está en la base de la desigualdad de las relaciones sociales de género, es necesario analizar las múltiples manifestaciones que se expresan en la menor posibilidad de las mujeres para incorporarse al mercado de trabajo remunerado, ejercer sus derechos, resolver las demandas de cuidado y poder construir una trayectoria laboral en igualdad de posibilidades que sus pares varones.

Para ello resulta fundamental contar con información confiable y sistemática, a lo cual se le suma la necesidad de que dicha información sea elaborada con un enfoque de género, entre otros, en tanto no existe neutralidad en las políticas públicas y por ende el análisis de género busca precisamente identificar las asimetrías de poder imperantes y como estas se traducen en las formas que adoptan los comportamientos laborales y la división sexual del trabajo. Si bien se han producido avances en las formas de recolección de información al interior del aparato estatal ${ }^{1}$, el Instituto Nacional de Estadísticas y Censos (INDEC) sigue sufriendo una intervención política de varios

1 Cabe destacar la creación del Observatorio de Género del Ministerio de Trabajo, Empleo y Seguridad Social, al igual que el diseño del Boletín de Seguridad Social que incorpora la desagregación de datos por sexo, una práctica que no estaba incorporada anteriormente y que implica un salto cualitativo fundamental para el análisis y la toma de decisiones, diseño y evaluación de las políticas laborales. 
años que ha limado la base de producción y confiabilidad del sistema estadístico y que a pesar de las reiteradas denuncias y acciones desarrolladas sigue su curso, perpetuando aún más la debilidad estadística y de las políticas públicas correctivas de las desigualdades existentes. De este modo, en la medida que no se avance en la introducción de cambios que permitan darle visibilidad a las múltiples discriminaciones existentes, no se podrán diseñar políticas públicas equitativas y eficaces, al mismo tiempo es un imperativo que se normalice la situación del Instituto.

\section{Gráfico 1}

Tasa de Actividad y Empleo femenina y brecha de género en la tasa de actividad, empleo y desocupación. Personas de 14 a 64 años. Total Aglomerados: 1990-2010

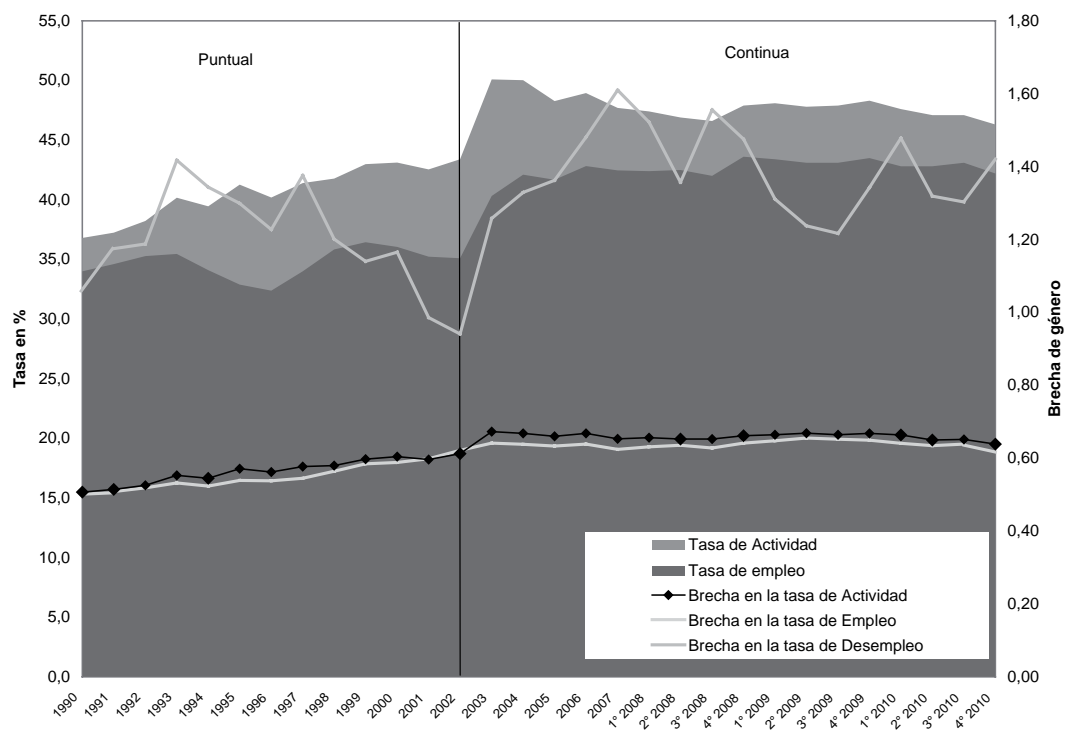

Fuente: Elaboración propia de Pautassi et al (2011) sobre la base del Boletín de Estadísticas de Género y Mercado de Trabajo. Observatorio de Empleo y Dinámica Empresarial, MTEySS, en base EPH (INDEC). 
Hecha esta salvedad en relación con la disponibilidad de información, en el gráfico ${ }^{2}$ anterior se comprueban las tendencias adelantadas: la tasa de actividad femenina se ha incrementado notablemente en Argentina, situación que condice con la propensión en toda América Latina, sin embargo este aumento no se ha traducido en una disminución de las desigualdades de género. Por el contrario, los datos recientes muestran un incremento de la brecha, dato que resulta llamativo y preocupante a la vez porque se produce en momentos de crecimiento económico como el que ha operado en la Argentina desde el 2003 en adelante, sin que el consiguiente incremento del empleo haya permitido reducir la brecha de género identificada.

El propio Ministerio de Trabajo, Empleo y Seguridad Social señala que la participación de la mujer en el empleo industrial se mantuvo reducida y no se evidencian cambios significativos desde la salida de la convertibilidad, inclusive en aquellas ramas intensivas en conocimiento, la tendencia todavía es incipiente, reafirmando que, aunque con diferentes matices, "las desigualdades de género están presentes en la mayoría de los sectores económicos. La estrategia del gobierno argentino en los últimos años ha sido ubicar al empleo decente en el centro del modelo de desarrollo económico, para recuperar la centralidad del trabajo como eje estructurante de la ciudadanía (...) Sin embargo, una de las asignaturas pendientes en la agenda pública es la equidad de género en el mercado de trabajo, donde las mujeres participan en una menor proporción que los varones, presentan tasas de desempleo más elevadas y acceden en menor medida al empleo, mostrando además, un mayor grado de precariedad laboral e ingresos laborales menores que los varones" (MTEySS, 2009).

De este modo, se comprueba que en un contexto de crecimiento económico no sólo no se ha revertido la desigualdad, sino que la misma se ha incrementado, ya que la disminución de la participación de la mujer en el mercado de trabajo ha sido un proceso continuo en los últimos años, así como el consecuente incremento de la desigualdad

2 En este apartado se incluye información procesada en Pautassi (et al, 2011). 
de género, con un aumento en la brecha especialmente en el caso de los grupos más jóvenes y con menor nivel educativo.

\section{Cuadro 1}

Tasa de Actividad femenina y brecha de género según nivel educativo y grupos de edad. Personas de 14 a 64 años. Total Aglomerados. 2003-2010.

\begin{tabular}{|c|c|c|c|c|c|c|c|c|c|c|c|c|}
\hline \multirow[b]{3}{*}{ 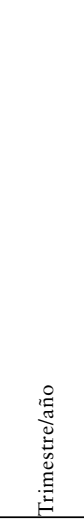 } & \multicolumn{6}{|c|}{ Tasa de participación en el mercado de trabajo } & \multicolumn{6}{|c|}{ Brecha de género } \\
\hline & \multirow[b]{2}{*}{ 苞 } & \multicolumn{2}{|c|}{$\begin{array}{l}\text { Grupos de } \\
\text { edad }\end{array}$} & \multicolumn{3}{|c|}{$\begin{array}{l}\text { Máximo Nivel Educativo } \\
\text { Alcanzado }\end{array}$} & \multirow[b]{2}{*}{ 苞 } & \multicolumn{2}{|c|}{$\begin{array}{l}\text { Grupos de } \\
\text { edad }\end{array}$} & \multicolumn{3}{|c|}{$\begin{array}{l}\text { Máximo Nivel } \\
\text { Educativo Alcanzado }\end{array}$} \\
\hline & & 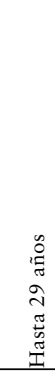 & 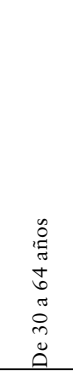 & 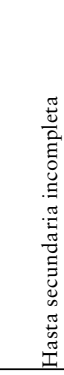 & 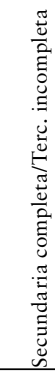 & 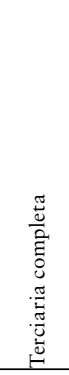 & & 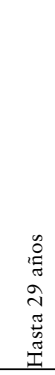 & 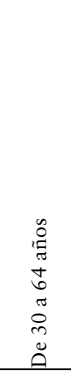 & 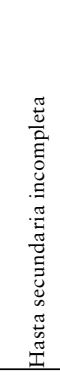 & 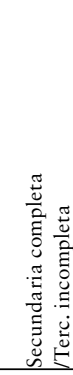 & 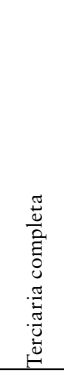 \\
\hline $3^{\circ} 2003$ & 58,7 & 50,6 & 64,4 & 48,5 & 61,5 & 86,4 & 0,73 & 0,78 & 0,70 & 0,63 & 0,74 & 0,91 \\
\hline $4^{\circ} 2003$ & 57,0 & 50,1 & 62,0 & 47,1 & 60,1 & 84,0 & 0,70 & 0,76 & 0,66 & 0,60 & 0,71 & 0,87 \\
\hline $1^{\circ} 2004$ & 56,9 & 49,2 & 62,3 & 47,4 & 59,9 & 84,5 & 0,70 & 0,76 & 0,67 & 0,61 & 0,73 & 0,88 \\
\hline $2^{\circ} 2004$ & 57,8 & 49,1 & 64,0 & 47,7 & 61,3 & 85,4 & 0,71 & 0,75 & 0,69 & 0,61 & 0,74 & 0,89 \\
\hline $3^{\circ} 2004$ & 57,6 & 48,9 & 63,9 & 46,9 & 61,2 & 85,7 & 0,71 & 0,74 & 0,69 & 0,60 & 0,73 & 0,90 \\
\hline $4^{\circ} 2004$ & 57,0 & 47,9 & 63,6 & 47,3 & 59,8 & 83,8 & 0,70 & 0,72 & 0,68 & 0,60 & 0,72 & 0,87 \\
\hline $1^{\circ} 2005$ & 55,7 & 46,8 & 62,0 & 46,3 & 58,1 & 82,9 & 0,68 & 0,72 & 0,67 & 0,59 & 0,71 & 0,87 \\
\hline $2^{\circ} 2005$ & 56,0 & 47,2 & 62,2 & 44,6 & 60,2 & 85,1 & 0,69 & 0,74 & 0,67 & 0,58 & 0,73 & 0,88 \\
\hline $3^{\circ} 2005$ & 56,4 & 46,5 & 63,3 & 45,2 & 59,8 & 85,3 & 0,69 & 0,69 & 0,68 & 0,58 & 0,71 & 0,88 \\
\hline $4^{\circ} 2005$ & 56,9 & 46,9 & 63,9 & 45,2 & 60,0 & 87,0 & 0,70 & 0,72 & 0,69 & 0,59 & 0,72 & 0,90 \\
\hline $1^{\circ} 2006$ & 57,0 & 47,4 & 63,5 & 46,1 & 59,3 & 86,0 & 0,70 & 0,74 & 0,68 & 0,60 & 0,71 & 0,89 \\
\hline $2^{\circ} 2006$ & 57,6 & 47,5 & 64,6 & 46,2 & 60,3 & 86,8 & 0,71 & 0,73 & 0,69 & 0,60 & 0,73 & 0,90 \\
\hline $3^{\circ} 2006$ & 56,9 & 47,3 & 63,5 & 44,1 & 61,2 & 85,0 & 0,69 & 0,72 & 0,68 & 0,57 & 0,72 & 0,88 \\
\hline $4^{\circ} 2006$ & 56,5 & 47,0 & 62,8 & 43,6 & 60,6 & 85,6 & 0,70 & 0,74 & 0,68 & 0,58 & 0,72 & 0,89 \\
\hline $1^{\circ} 2007$ & 56,2 & 47,0 & 62,3 & 44,0 & 59,5 & 84,0 & 0,69 & 0,72 & 0,67 & 0,57 & 0,72 & 0,86 \\
\hline $2^{\circ} 2007$ & 55,9 & 44,9 & 63,3 & 42,2 & 60,0 & 85,8 & 0,68 & 0,69 & 0,68 & 0,55 & 0,71 & 0,89 \\
\hline $4^{\circ} 2007$ & 54,8 & 46,0 & 60,6 & 41,4 & 58,9 & 84,4 & 0,68 & 0,74 & 0,65 & 0,56 & 0,71 & 0,87 \\
\hline
\end{tabular}




\begin{tabular}{|l|l|l|l|l|l|l|l|l|l|l|l|l|}
$1^{\circ} 2008$ & 55,6 & 46,0 & 62,0 & 42,7 & 58,7 & 83,4 & 0,69 & 0,74 & 0,67 & 0,57 & 0,71 & 0,86 \\
$2^{\circ} 2008$ & 55,1 & 43,6 & 62,4 & 41,4 & 58,8 & 84,8 & 0,68 & 0,70 & 0,67 & 0,55 & 0,71 & 0,87 \\
$3^{\circ} 2008$ & 54,6 & 44,8 & 61,0 & 41,2 & 58,6 & 84,0 & 0,68 & 0,72 & 0,66 & 0,56 & 0,70 & 0,88 \\
$4^{\circ} 2008$ & 56,0 & 45,1 & 63,0 & 42,4 & 58,8 & 86,5 & 0,70 & 0,72 & 0,68 & 0,57 & 0,70 & 0,90 \\
$1^{\circ} 2009$ & 56,2 & 44,9 & 63,6 & 42,3 & 59,3 & 85,6 & 0,70 & 0,72 & 0,69 & 0,56 & 0,72 & 0,89 \\
$2^{\circ} 2009$ & 56,0 & 45,3 & 62,6 & 41,6 & 59,8 & 84,8 & 0,70 & 0,72 & 0,68 & 0,56 & 0,72 & 0,89 \\
$3^{\circ} 2009$ & 55,8 & 44,0 & 63,0 & 41,5 & 59,3 & 85,7 & 0,69 & 0,70 & 0,68 & 0,56 & 0,70 & 0,91 \\
$4^{\circ} 2009$ & 56,2 & 42,8 & 64,5 & 41,5 & 60,1 & 85,6 & 0,70 & 0,69 & 0,69 & 0,55 & 0,72 & 0,89 \\
$1^{\circ} 2010$ & 55,7 & 43,4 & 63,4 & 41,2 & 58,8 & 84,1 & 0,70 & 0,70 & 0,69 & 0,56 & 0,71 & 0,88 \\
$2^{\circ} 2010$ & 54,8 & 43,5 & 61,9 & 40,2 & 57,4 & 85,5 & 0,68 & 0,69 & 0,67 & 0,54 & 0,68 & 0,89 \\
$3^{\circ} 2010$ & 55,1 & 42,8 & 62,8 & 40,7 & 58,2 & 84,2 & 0,69 & 0,70 & 0,68 & 0,56 & 0,68 & 0,88 \\
$4^{\circ} 2010$ & 54,4 & 41,7 & 62,4 & 38,5 & 58,3 & 84,4 & 0,68 & 0,67 & 0,67 & 0,52 & 0,69 & 0,87 \\
\hline
\end{tabular}

Fuente: Elaboración propia de Pautassi et al (2011) sobre la base del Boletín de Estadísticas de Género y Mercado de Trabajo. Observatorio de Empleo y Dinámica Empresarial, DGEyEL, SSPTyEL, MTEySS, en base a la EPH del INDEC.

A su vez, y debido a que la Argentina es un país federal, el comportamiento del empleo femenino presenta importantes heterogeneidades regionales (Cuadro 2). Así se observa que en el año 2010 la tasa de actividad disminuye en mujeres que viven en hogares con presencia de menores, en los hogares más pobres y entre aquellas con menor nivel educativo, dando cuenta de un comportamiento estratificado y altamente dependiente de las responsabilidades de cuidado. A su vez, la brecha entre mujeres es menor cuando se considera la tasa de empleo, lo cual se explica porque aquellas mujeres en situación de vulnerabilidad encuentran mayores dificultades para insertarse en ocupaciones remuneradas y no se han diseñado políticas laborales que apunten a revertir las brechas. 


\section{Cuadro 2}

Tasa de actividad, empleo y desempleo de mujeres de 19 a 49 años. Año 2010.

Nota: los números en negrita son inferiores a los valores promedio.

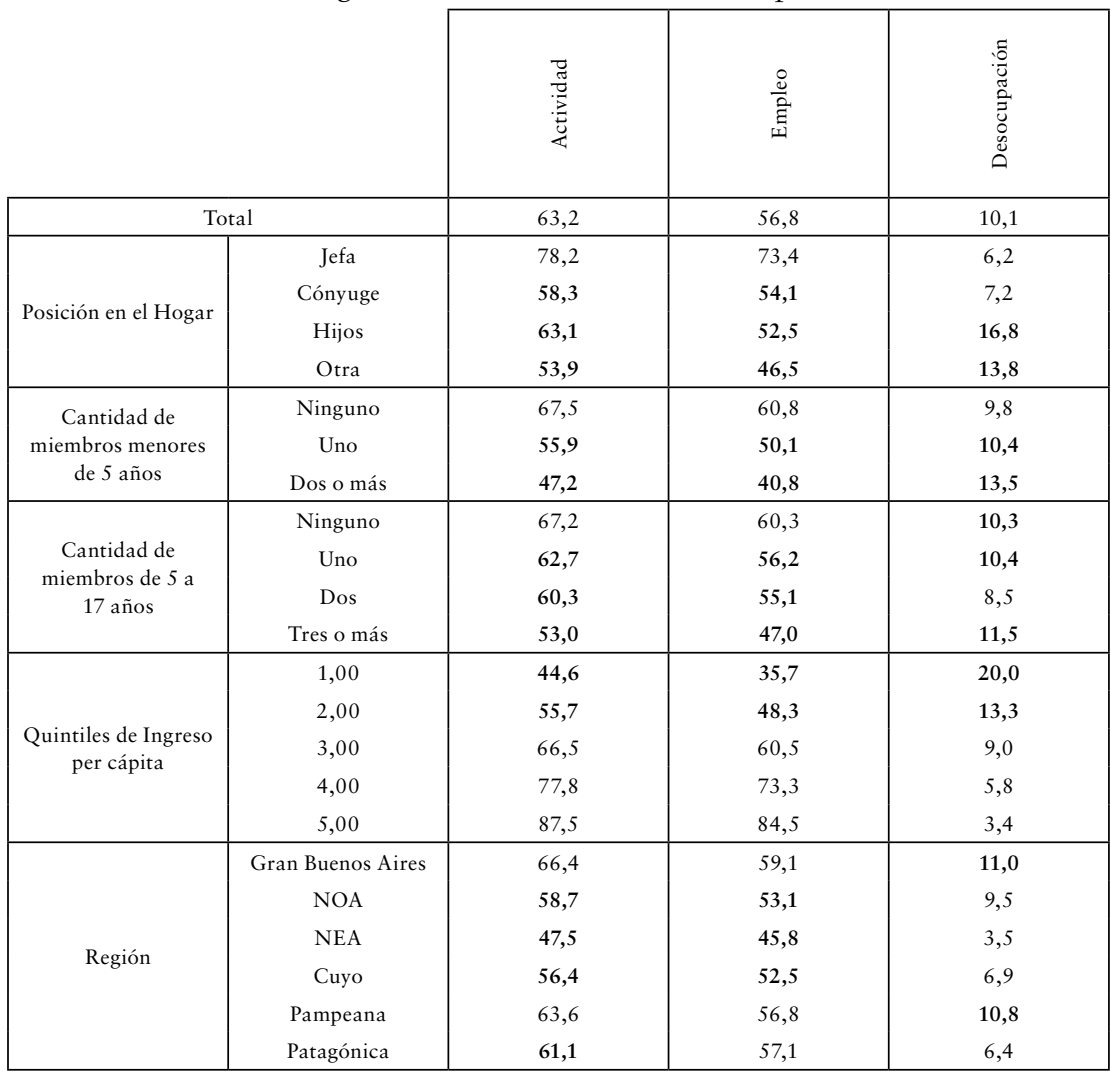

Fuente: Elaboración propia de Pautassi et al (2011) sobre la base de datos de EPH. INDEC.

Como hemos visto anteriormente, en los últimos años se revierte la tendencia al incremento de la proporción de empleo asalariado sin descuento jubilatorio, por lo que el número de aportantes ha aumentado significativamente, dando cuenta también de una decisión de la política de empleo de avanzar en disminuir el empleo no registrado. No obstante, poco se ha avanzado en una mayor inserción de las 
mujeres en el empleo asalariado privado formal, ya que las brechas señaladas no han estado en el eje del accionar gubernamental. Como podemos comprobar en el siguiente gráfico, cerca de 7 de cada 10 empleos en este sector es ocupado por varones. No se observan cambios significativos de esta tendencia, como así tampoco de la segmentación del trabajo, que conlleva a una baja participación de la mujer en la industria y una fuerte concentración del empleo femenino en servicios.

\section{Grafico 2}

Composición del empleo asalariado privado registrado femenino y participación de la mujer en el total de puestos de trabajo registrados. Total país.

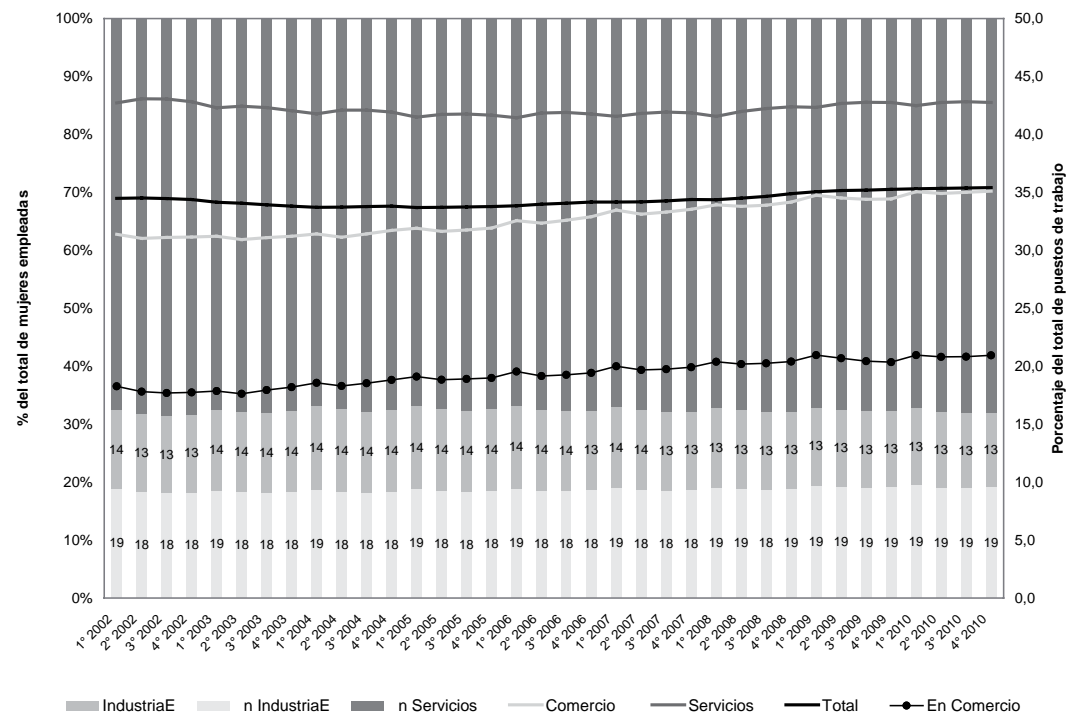

Fuente: Elaboración propia de Pautassi et al (2011) sobre la base del Boletín de Estadísticas de Género y Mercado de Trabajo, Observatorio de Empleo y Dinámica Empresarial, DGEyEL, SSPTyEL, MTEySS, en base a SIPA. 
El nivel de segmentación es creciente cuando se observa la composición del empleo en cada sector. Por ejemplo, en la industria el $18.9 \%$ de los puestos de trabajo son ocupados por mujeres, en este sector la proporción de mujeres varía desde un mínimo de $6 \%$ en "metales" a un máximo de $58 \%$ en "confecciones". La misma heterogeneidad se observa en servicios, donde el $42.8 \%$ de los puestos lo ocupan mujeres, aunque en transporte esta proporción desciende a $5.4 \%$ y alcanza un máximo de $71.4 \%$ en "servicios sociales y de salud" (MTEySS, 2010) dando cuenta de comportamientos que caracterizan a un mercado de trabajo donde las "ocupaciones típicamente femeninas" siguen liderando la inserción sectorial de las mujeres y donde desde la política pública no se ha estimulado otro tipo de inserción ocupacional.

A tal punto se produce esta segmentación que analizando la población de 19 a 49 años de edad, en base a datos de la Encuesta Permanente de Hogares (EPH) se constata que las mujeres de estas edades que se incorporan al empleo asalariado formal o como cuenta propia profesionales o empleadoras (que podría suponer cierta formalidad) representan el 33\% del total de mujeres. Este porcentaje desciende a 19\% entre aquellas que viven en hogares con dos o más niños, niñas y adolescentes en el hogar (Cuadro 3).

\section{Cuadro 3}

Composición de la población de 19 a 49 años según condición de actividad, categoría ocupación por número de niños menores de 5 años en el hogar. Año 2010. (Página siguiente) 


\begin{tabular}{|c|c|c|c|c|c|}
\hline Sexo & $\begin{array}{l}\text { Condición } \\
\text { de actividad/ } \\
\text { Categoría } \\
\text { ocupacional }\end{array}$ & Ninguno & Uno & Dos o más & Total \\
\hline \multirow{8}{*}{ Varón } & Inactivo & 13,0 & 4,7 & 5,4 & 10,6 \\
\hline & Desocupado & 6,2 & 5,8 & 5,0 & 6,1 \\
\hline & $\begin{array}{l}\text { Asalariado } \\
\text { no registrado, } \\
\text { trabajador } \\
\text { familiar }\end{array}$ & 18,0 & 21,0 & 26,1 & 19,2 \\
\hline & $\begin{array}{l}\text { Cuenta propia } \\
\text { operativa o no } \\
\text { calificada }\end{array}$ & 10,6 & 11,9 & 13,1 & 11,0 \\
\hline & $\begin{array}{c}\text { Servicio } \\
\text { doméstico en los } \\
\text { hogares }\end{array}$ & 0,1 & 0,1 & 0,1 & 0,1 \\
\hline & $\begin{array}{l}\text { Asalariado } \\
\text { registrado }\end{array}$ & 45,5 & 50,2 & 43,6 & 46,5 \\
\hline & $\begin{array}{l}\text { Empleador } \\
\text { cuentapropia } \\
\text { profesional o } \\
\text { calificado }\end{array}$ & 6,5 & 6,3 & 6,7 & 6,5 \\
\hline & Total & 100,0 & 100,0 & 100,0 & 100,0 \\
\hline \multirow{8}{*}{ Mujer } & Inactiva & 32,5 & 44,1 & 52,8 & 36,8 \\
\hline & Desocupada & 6,6 & 5,8 & 6,4 & 6,4 \\
\hline & $\begin{array}{c}\text { Asalariada } \\
\text { no registrada, } \\
\text { trabajadora } \\
\text { familiar }\end{array}$ & 9,8 & 8,9 & 9,9 & 9,6 \\
\hline & $\begin{array}{l}\text { Cuenta propia } \\
\text { operativa o no } \\
\text { calificada }\end{array}$ & 4,9 & 5,4 & 4,5 & 5,0 \\
\hline & $\begin{array}{c}\text { Servicio } \\
\text { doméstico en los } \\
\text { hogares }\end{array}$ & 9,4 & 8,1 & 7,3 & 8,9 \\
\hline & $\begin{array}{l}\text { Asalariada } \\
\text { registrada }\end{array}$ & 32,6 & 24,4 & 15,2 & 29,3 \\
\hline & $\begin{array}{c}\text { Empleadora } \\
\text { cuentapropia } \\
\text { profesional o } \\
\text { calificada }\end{array}$ & 4,2 & 3,3 & 3,9 & 3,9 \\
\hline & Total & 100,0 & 100,0 & 100,0 & 100,0 \\
\hline
\end{tabular}


Si bien el nivel actual de informalidad muestra los resultados de la disminución de los últimos años, aún afecta a importante cantidad de personas, y en forma diferenciada a mujeres y varones, situación que debe ser analizada a la luz de la dinámica demográfica. En Argentina, al igual que en América Latina, se presenta un proceso de envejecimiento que está acompañado por dos características preocupantes: en primer lugar, se está produciendo -y continuará haciéndolo- a un ritmo más rápido que el que se observó en los países centrales, y en segundo lugar, dicho incremento de los y las mayores está enmarcado en un contexto de altos niveles de pobreza, baja cobertura en seguridad social, condiciones de salud inequitativas y una fuerte presión sobre las familias en todos los aspectos de cuidado. Por ende, la urgencia por aumentar los niveles de registración en el empleo son claros y demandan una dinámica de previsión social actual que anticipe los riesgos que la pasividad laboral van a acarrear a quienes desarrollan una trayectoria laboral desprotegida, lo cual es aún más severa, para aquellas mujeres a cargo de tareas de cuidado exclusivamente.

En síntesis, la evidencia disponible no sólo muestra que las mujeres tienen mayor presencia en puestos de baja calidad, entendidos como aquellos con horas de trabajo insuficientes, sin acceso a seguridad social y con salarios menores por acción de la discriminación salarial, sino que estas desigualdades también están presentes en la trayectoria laboral, la cual va a estar permeada permanentemente por las responsabilidades de cuidado y por el uso asimétrico del tiempo. Sin embargo, en la agenda pública no se encuentran estos temas en debate, sino que la tendencia durante la década pasada de las políticas públicas fue de debilitamiento de las políticas sociales, transfiriendo al hogar y al mercado gran parte de las tareas de cuidado y dentro del hogar directamente a las mujeres, sin que se asuma la responsabilidad de dicha transferencia. 


\section{Tiempo y cuidado: dimensiones ausentes en la política pública}

En la misma línea argumentativa, el examen del comportamiento del mercado de trabajo remunerado no debe circunscribirse solamente a las variables de análisis disponibles, sino que se debe considerar las formas en que las mujeres se insertan, los diferenciales de calidad en la ocupación, cobertura en seguridad social y las trayectorias laborales que pueden desarrollar en conjunción con el análisis de la distribución del tiempo total de trabajo y las responsabilidades de cuidado.

Por cuidado se entiende al conjunto de actividades necesarias para satisfacer las necesidades mínimas para la existencia y reproducción de las personas, que las "nutren" en el sentido de otorgarles los elementos físicos y simbólicos que les permiten vivir una vida plena y en sociedad, y que incluye tanto el brindar directamente cuidado y atención a las personas dependientes, como son los niños, niñas y adolescentes; las personas adultas mayores, enfermas o con discapacidades y la provisión de las precondiciones para que ese cuidado pueda realizarse ${ }^{3}$.

Precisamente el ámbito del cuidado es el que tradicionalmente ha sido delegado en las mujeres, dejando en evidencia no solo la conformación del modelo patriarcal que ha asignado este lugar a las mujeres, sino como la organización social del cuidado en las sociedades latinoamericanas es una fuente de desigualdad social y de género e, incluso, de reproducción de la pobreza (CEPAL, 2009). Esta debilidad de las políticas públicas se traduce por lo tanto en un incremento de la desigualdad, los hogares que disponen de recursos pueden adquirir los servicios en el sector privado, quienes no cuentan con dinero, en cambio, deben diseñar estrategias al interior del hogar y eso condiciona la disponibilidad de tiempo para realizar tareas remuneradas. En otras palabras, quienes menos tienen, verán mermadas sus posibilidades de acceder a servicios de cuidado porque deben adquirirlos en el mercado ante la falta de provisión estatal. Es decir,

3 Al respecto ver Batthyány (2004) y Rico (2005), entre otros. 
la baja presencia del Estado en la satisfacción de las necesidades y demandas de la población, evidencia que resulta urgente modificar la lógica de las políticas sociales imperantes. Esto significa, que tal como Marco y Rico (2013) señalan, las políticas actuales -de seguridad social, educación y salud, ya sean de carácter contributivo o no contributivo-, deben necesariamente ser reorientadas de manera de superar las brechas existentes y se debe considerar la posibilidad de incluir un "cuarto pilar": el cuidado de todos y todas y la atención a las personas con algún nivel de dependencia.

A su vez, y dando cuenta de los círculos de inequidad que se generan, por una parte, la ausencia de infraestructura, servicios y regulaciones de cuidado afecta a las mujeres y por la otra limitan las posibilidades de desarrollo de aptitudes y habilidades necesarias de los niños, niñas y adolescentes para el desempeño de la carrera escolar en hogares con menores niveles de ingreso. En otros términos, la desigualdad actual no sólo afecta el derecho al cuidado de los niños y niñas, sino que impacta, especialmente en los hogares con menores ingresos, debido a que las mujeres encuentran condicionada su disponibilidad de tiempo para incorporarse en el mercado de trabajo, debiendo desarrollar las mujeres en la mayoría de los casos, estrategias de cuidado familiar, que limitan la cantidad de horas disponibles, condicionan la elección de la forma de inserción y por lo tanto impactan en la situación de pobreza de los hogares.

En general, las disposiciones que se presentan en relación con el cuidado se las puede agrupar en dos instancias: i) normas y políticas vinculadas a organizar el "cuidado" de los miembros del hogar y personas bajo responsabilidad de las trabajadoras asalariadas, ii) políticas sociales dirigidas a la protección de los propios "cuidados", por caso, sistema educativo para niños y jóvenes; sistema de salud para las personas de la tercera edad, programas de salud maternoinfantiles, entre otros.

En ambos casos, desde la normativa y la lógica de tratamiento, no se observa que el cuidado en todas sus dimensiones sea considerado el eje de la regulación y de las políticas sino precisamente es calificado, por un lado, como herramienta para la inserción de la mujer en 
el empleo remunerado, con total prescindencia de la responsabilidad paterna; por el otro, como parte de programas asistenciales en la esfera de ministerios sectoriales. Esto es, se lo considera "vinculado con" o en "relación a" pero no existe, salvo excepciones, un tratamiento del tema de manera autónoma y en toda su amplitud y complejidad (Pautassi, 2007).

A pesar de la profusa normativa reformista durante los años noventa, perduran las regulaciones de la Ley de Contrato de Trabajo que contemplan un título especial "Del trabajo de las mujeres" (artículo 172 a 186 Ley de Contrato de Trabajo, LCT) ${ }^{4}$ junto con el de "menores" que garantiza la estabilidad en el empleo a toda mujer durante la gestación. Esta garantía opera como derecho adquirido a partir del momento que la mujer notifique fehacientemente al empleador la fecha presunta del parto (artículo 177, LCT) y se establece una presunción de despido por causa de embarazo, cuando el mismo fuese dispuesto dentro del plazo de siete meses y medio anteriores o posteriores a la fecha de parto, siempre que la mujer haya cumplido con su obligación de notificar la fecha presunta del nacimiento, no pudiendo efectuar esta notificación con anterioridad o posterioridad a los plazos señalados. En tal caso, se impone una indemnización agravada para tal supuesto, equivalente a un año de remuneraciones, que se acumulará a la indemnización por despido sin justa causa (artículo 178 LCT). La licencia por maternidad se mantiene en 90 días para las trabajadoras en relación de dependencia reguladas ( 30 días con anterioridad al parto y 60 días posteriores), mientras que a nivel del empleo público, recientes modificaciones como la ocurrida en la provincia de Córdoba, extienden el beneficio a 180 días; en otras provincias alcanza los 110 días y algunos regímenes especiales, como la Universidad Nacional de General Sarmiento se ha extendido recientemente a 180 días. En relación con el tiempo de cuidado para

4 Ley 20744 "Contrato de Trabajo" de 1974 (texto ordenado Decreto 390 de 1976), la ley 24013 de 1991; 24465; 24467 y 24557 de 1995 ; ley 25013 de 1998 y 25250 de 2000 que fue derogada por ley 25877 de 2004 , ley 26.088 de 2006 y reformas posteriores particularmente referidas a emergencia pública (económica y social). El ámbito de aplicación de la LCT comprende a los trabajadores en relación de dependencia, con excepción de los dependientes de la administración pública Nacional, Provincial y Municipal, los trabajadores del servicio doméstico y los trabajadores agrarios que se rigen por Estatutos especiales. 
los padres, la LCT solo considera dos días por nacimiento. En los últimos años, se presentaron proyectos de ley que lo extendían a 15 y otros a cinco días (que cuenta con aprobación del Senado) con la equiparación entre nacimiento y adopción, aunque todavía no alcanzan rango legal (Pautassi y Rico, 2011:6).

Nuevamente en el sector público es donde se han producido mayores avances, en la reforma de la provincia de Córdoba, se extendió a ocho días la licencia para los padres que trabajan en el ámbito estatal; y en municipios como Rosario han extendido el beneficio a diez días hábiles para sus empleados varones y el municipio de Morón lo extendió a 20 días hábiles en el año 2010. El resto del ciclo vital no cuenta con disponibilidad de tiempo para asumir el cuidado, ni de niños, niñas y adolescentes como tampoco de adultos mayores o enfermos bajo responsabilidad del hogar. La excepción es el caso de nacimiento de niños o niñas con síndrome de down, que la licencia se extiende a seis meses. La lactancia se extiende en una hora diaria dividida en dos medias horas durante el primer año de vida del niño, niña y adolescente. Claro está, que estas licencias son sólo para trabajadoras asalariadas, quedando excluidas el universo de trabajadoras informales o cuentapropistas.

Desde la política social, las ausencias son múltiples y significativas. Para comenzar, la disponibilidad de datos es escasísima, y no solamente considerando indicadores más complejos como medición del uso del tiempo, sino tampoco se recaba información referida, por caso, a cuidado infantil y mucho menos para enfermos o cuidado de personas adultas mayores. El Censo Nacional de Población y la Encuesta Permanente de Hogares (EHP) incluyen en su cuestionario la preguntan sobre asistencia escolar pero recién a partir de los 3 años, dejando sin indagar la asistencia a guarderías y centros de cuidado infantil. Sólo algunos relevamientos, como la Encuesta Nacional de Actividades de Niños y Adolescentes (2004) y la Encuesta de Condiciones de Vida (2001) incorporaron algunas preguntas sobre asistencia de menores de tres años, pero a la fecha ninguna tiene garantizada la continuidad en el sistema estadístico nacional. En el caso de la principal fuente de información sobre el comportamiento 
del mercado laboral que es la EPH, interroga sobre un conjunto de beneficios laborales para los asalariados, pero no incorpora ninguna pregunta sobre guarderías, lo cual deja no solo un vacío en relación con la información, sino también la posibilidad de conocer los grados de cumplimiento de los empleadores de esta obligación. A su vez, tampoco se cumplen con los postulados establecidos en la Ley de educación nacional (ley 26206/06) que establece la obligatoriedad en la recolección de información de acciones en materia de cuidado infantil ${ }^{5}$.

En consecuencia, y con el objetivo de poder avanzar en identificar cómo se resuelve el cuidado en los aglomerados urbanos en Argentina, se realizaron otros estudios secundarios y encuestas que buscan dar cuenta de las formas en que se resuelve el cuidado en los hogares en Argentina.

Según lo confirma una encuesta realizada por el Equipo Latinoamericano de Justicia y Género (ELA) en el área metropolitana de Buenos Aires ${ }^{6}$, que busca indagar las formas de resolución del cuidado de niños, niñas y adolescentes menores de 13 años, en una semana típica cuando los niños están en el hogar, las madres son las principales responsables de cuidar: el $76 \%$ de los casos se encargan del cuidado y en el $50 \%$ de los casos lo hacen, declarativamente, de manera exclusiva. Las tareas de cuidado recaen significativamente en menor medida en los padres $(22 \%)$ y en otras personas mayores de edad, ya sea que vivan o no en el hogar de referencia (18\% y $10 \%$ respectivamente). En ambos casos se trata mayoritariamente de abuelas y, en menor medida, de hermanos/as y tías. Sólo el 5\% de los hogares

5 La Encuesta Nacional de Protección Social (ENAPROSS), implementada por el Ministerio de Trabajo en el año 2010 en algunos aglomerados del país incluyó un módulo sobre cuidado infantil, pero aún no están disponibles sus resultados. Debido a que el país carece de un plan plurianual de encuestas tampoco se tiene información sobre si la misma tiene prevista una continuidad en el tiempo.

6 En base a un estudio de opinión exploratorio basado en una encuesta a varones y mujeres entre 18 y 65 años de edad, residentes en el Área Metropolitana de Buenos Aires, en base a un cuestionario semi estructurado aplicado en el mes de agosto del 2011. Se efectuaron una totalidad de 804 entrevistas (654 telefónicas y 150 presenciales). El total de hogares fue de 750 casos y se efectuó una sobre-muestra de hogares con niños menores de 13 años: 54 casos con la finalidad de obtener mayor confiabilidad en la información sobre el trabajo de cuidado que efectúan las familias. Los datos fueron ponderados por zona: Ciudad Autónoma de Buenos Aires (CABA), y Gran Buenos Aires (GBA); sexo y nivel educativo según parámetros poblacionales. 
relevados contrata personal para el cuidado de los niños, de los cuales en el 3.3\% de los casos se trata de la misma persona contratada para las tareas domésticas (limpieza, cocina).

\section{Gráfico 3}

Principales responsables del cuidado en el hogar

Base: total hogares con menores de 13 años $(n=395)$

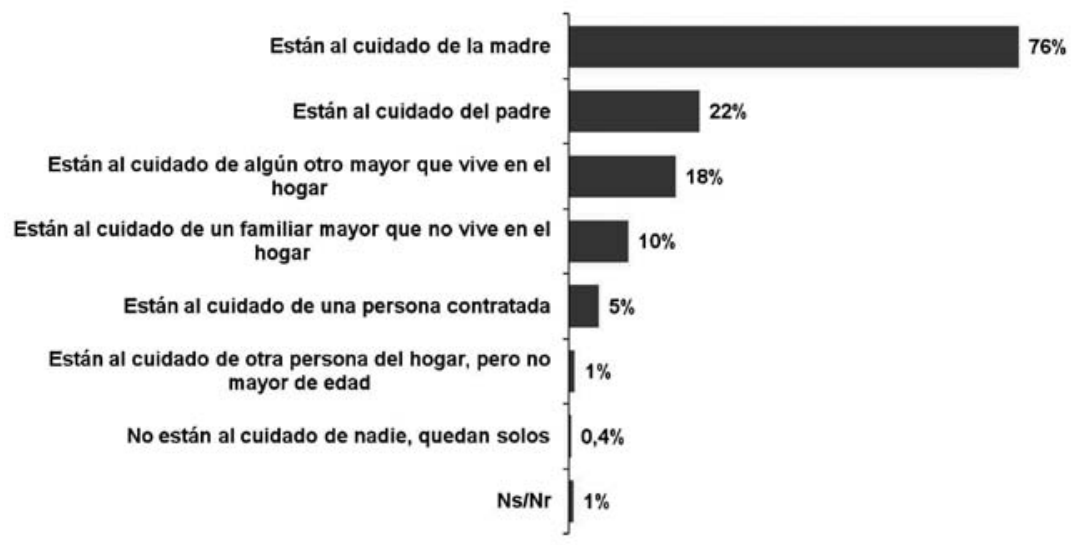

Fuente: ELA, 2012.

En relación con la asistencia de los niños y niñas más pequeños hay que diferenciar el caso de las guarderías o jardines maternales (de 0 a 2 años) de los jardín de infantes (de 3 a 5 años), en tanto los últimos forman parte de manera integrada con el sistema educativo, mientras que las guarderías se ha establecido como función asistencial, desestimando su importancia pedagógica y su papel en la organización del cuidado en los hogares (Rodríguez Enríquez, 2010).

Según los datos arrojados por la encuesta de ELA (2012), el 87\% de los hogares con niños y niñas menores de 13 años todos o algunos de ellos está escolarizado. Este porcentaje desciende a $31 \%$ en aquellos hogares en los que sólo hay niños menores de 3 años. En aquellos hogares en los que solo viven niños y niñas que se encuentran en edad 
para asistir a la escuela primaria, el 100\% asiste a la escuela independientemente del lugar de residencia. Por su parte, en los hogares en los que solo viven niños menores de tres años, casi el 70\% no concurren a la escuela, tanto en el caso de CABA como GBA. En cambio, en los hogares en los que solo hay niños entre 3 y 6 años se observan diferencias según zona de residencia. Mientras que en CABA todos concurren a establecimientos educativos sólo el $83 \%$ lo hace en GBA.

\section{Cuadro 4}

Concurrencia a la escuela según edad de niños y niñas - Datos según región

\begin{tabular}{|c|c|c|c|c|c|c|c|c|}
\hline & \multirow{2}{*}{$\begin{array}{c}\text { TOTAL } \\
\text { CABA }\end{array}$} & \multicolumn{3}{|c|}{$\mathrm{CABA}$} & \multirow{2}{*}{$\begin{array}{c}\text { TOTAL } \\
\text { GBA }\end{array}$} & \multicolumn{3}{|c|}{ GBA } \\
\hline & & \begin{tabular}{|c} 
Solo \\
menores de \\
3 años
\end{tabular} & $\begin{array}{l}\text { Solo niños } \\
\text { entre } 3 \text { y } 6 \\
\text { años }\end{array}$ & \begin{tabular}{|c|} 
Solo niños \\
entre 7 y 12 \\
años \\
\end{tabular} & & $\begin{array}{c}\text { Solo } \\
\text { menores de } \\
3 \text { años }\end{array}$ & $\begin{array}{l}\text { Solo niños } \\
\text { entre } 3 \text { y } 6 \\
\text { años }\end{array}$ & $\begin{array}{c}\text { Solo niños } \\
\text { entre } 7 \text { y } 12 \\
\text { años }\end{array}$ \\
\hline $\begin{array}{c}\text { Concurren } \\
\text { todos a la } \\
\text { escuela }\end{array}$ & $83 \%$ & $16 \%$ & $100 \%$ & $100 \%$ & $67 \%$ & $19 \%$ & $83 \%$ & $100 \%$ \\
\hline $\begin{array}{c}\text { Algunos } \\
\text { concurren y } \\
\text { otros no }\end{array}$ & $5 \%$ & $15 \%$ & - & - & $20 \%$ & $12 \%$ & $6 \%$ & - \\
\hline $\begin{array}{c}\text { Ninguno } \\
\text { concurre a la } \\
\text { escuela }\end{array}$ & $12 \%$ & $69 \%$ & - & - & $13 \%$ & $69 \%$ & $11 \%$ & - \\
\hline
\end{tabular}

Base: total hogares con menores de 13 años $(n=395)$.

Fuente. ELA, 2012.

El aumento de la asistencia escolar a partir de los 3 años de edad se vincula con diversos factores, entre los cuales se puede destacar los imaginarios y representaciones sociales que elaboran las familias en torno al momento adecuado para que sus hijos/as asistan a los establecimientos educativos, la extensión de la obligatoriedad al nivel inicial a partir de los 5 años -que ha tenido un efecto positivo en la cobertura y la asistencia- y la pretensión de universalización de la educación a partir de los 4 años de edad propuesto por la Ley de Educación Nacional. 
Sin embargo, y a pesar de estas garantías, existe para los primeros años del nivel inicial una oferta estatal residual y escasa tanto en CABA como en GBA. El límite al que se enfrenta la cobertura del sistema educativo en edades tempranas tiene importantes consecuencias para las familias de los estratos socioeconómicos más modestos (ELA, 2012). Y cabe recordar que estos datos corresponden al principal aglomerado urbano de la Argentina, lo cual si analiza las situaciones heterogéneas presentes a nivel provincial (Cuadro 5) se puede comprobar que, con excepción de la provincia de Misiones, en todas las jurisdicciones el sector estatal perdió peso en los últimos años, evidenciándose un incremento de cobertura del sector privado. Si bien en promedio ocurrió lo mismo en las salas de 3 y 4 años, en este universo la heterogeneidad jurisdiccional es mayor.

\section{Cuadro 5}

Nivel inicial. Salas de 3,4 y 5 años de educación común. Alumnos y proporción de matrícula atendida en el sector estatal. 2000-2010

\begin{tabular}{|c|c|c|c|c|c|c|c|c|c|c|c|c|}
\hline & \multicolumn{4}{|c|}{3 años } & \multicolumn{4}{|c|}{4 años } & \multicolumn{4}{|c|}{5 años } \\
\hline & \multicolumn{2}{|c|}{2000} & \multicolumn{2}{|c|}{2010} & \multicolumn{2}{|c|}{2000} & \multicolumn{2}{|c|}{2010} & \multicolumn{2}{|c|}{2000} & \multicolumn{2}{|c|}{2010} \\
\hline & $\begin{array}{c}\text { Total } \\
\text { alumnos }\end{array}$ & $\begin{array}{c}\% \\
\text { estatal }\end{array}$ & $\begin{array}{c}\text { Total } \\
\text { alumnos }\end{array}$ & 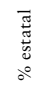 & $\begin{array}{c}\text { Total } \\
\text { alumnos }\end{array}$ & 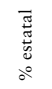 & $\begin{array}{c}\text { Total } \\
\text { alumnos }\end{array}$ & 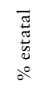 & $\begin{array}{c}\text { Total } \\
\text { alumnos }\end{array}$ & 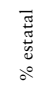 & $\begin{array}{c}\text { Total } \\
\text { alumnos }\end{array}$ &  \\
\hline Total País & 182.005 & 61,6 & 251.298 & 57,5 & 358.734 & 67,1 & 512.611 & 67,0 & 705.858 & 76,8 & 710.956 & 72,1 \\
\hline $\begin{array}{c}\text { Buenos } \\
\text { Aires }\end{array}$ & 134.219 & 66,2 & 164.073 & 59,2 & 184.155 & 65,0 & 220.900 & 60,7 & 238.032 & 66,2 & 252.592 & 61,2 \\
\hline Catamarca & 187 & 56,8 & 1.235 & 69,8 & 1.444 & 50,4 & 4.941 & 74,2 & 7.699 & 88,8 & 6.977 & 80,5 \\
\hline Chaco & 1.341 & 86,2 & 2.431 & 77,2 & 4.795 & 87,0 & 11.420 & 89,2 & 23.901 & 93,9 & 22.568 & 91,7 \\
\hline Chubut & 1.062 & 51,5 & 3.090 & 64,6 & 3.996 & 80,4 & 7.589 & 86,1 & 9.108 & 92,3 & 9.384 & 88,9 \\
\hline $\begin{array}{l}\text { Ciudad de } \\
\text { Bs. As. }\end{array}$ & 20.443 & 45,7 & 25.807 & 37,6 & 29.720 & 54,0 & 33.817 & 43,9 & 34.462 & 53,5 & 38.711 & 45,8 \\
\hline Córdoba & 1.328 & 11,8 & 8.901 & 63,8 & 28.358 & 77,5 & 46.811 & 74,5 & 55.845 & 78,5 & 59.631 & 74,2 \\
\hline Corrientes & 2.214 & 70,1 & 2.566 & 65,9 & 5.752 & 66,1 & 9.570 & 76,0 & 25.833 & 90,1 & 23.161 & 87,1 \\
\hline Entre Ríos & 1.160 & 35,7 & 3.819 & 59,0 & 7.897 & 56,3 & 14.445 & 66,1 & 25.338 & 77,3 & 23.635 & 73,2 \\
\hline Formosa & 360 & 8,3 & 908 & 37,6 & 2.099 & 53,3 & 5.763 & 79,8 & 12.303 & 89,4 & 11.496 & 87,1 \\
\hline Jujuy & 738 & 58,2 & 1.807 & 50,9 & 3.099 & 67,0 & 7.476 & 79,5 & 15.093 & 90,8 & 13.343 & 87,6 \\
\hline La Pampa & 99 & 0,0 & 300 & 25,0 & 618 & 42,4 & 2.452 & 74,5 & 5.906 & 91,5 & 5.671 & 90,5 \\
\hline
\end{tabular}




\begin{tabular}{|c|c|c|c|c|c|c|c|c|c|c|c|c|}
\hline La Rioja & 978 & 70,2 & 1.818 & 73,5 & 3.970 & 86,8 & 5.679 & 86,5 & 7.648 & 92,3 & 6.590 & 88,1 \\
\hline Mendoza & 885 & 4,6 & 1.289 & 22,3 & 3.385 & 42,1 & 20.638 & 75,3 & 33.997 & 86,3 & 32.149 & 82,1 \\
\hline Misiones & 453 & 61,3 & 1.037 & 27,0 & 2.526 & 43,6 & 10.682 & 71,1 & 25.905 & 81,0 & 25.001 & 82,7 \\
\hline Neuquén & 1.168 & 66,6 & 1.593 & 70,9 & 5.361 & 86,6 & 6.756 & 84,6 & 10.860 & 90,8 & 10.556 & 87,7 \\
\hline Río Negro & 848 & 37,5 & 2.322 & 66,7 & 5.982 & 80,9 & 8.511 & 82,3 & 12.346 & 84,3 & 11.456 & 81,0 \\
\hline Salta & 749 & 63,7 & 1.912 & 74,4 & 4.220 & 68,1 & 11.816 & 80,4 & 27.790 & 89,0 & 26.490 & 83,5 \\
\hline San Juan & 31 & 28,9 & 971 & 33,6 & 2.618 & 42,1 & 6.954 & 61,1 & 15.187 & 86,3 & 15.385 & 82,2 \\
\hline San Luis & 506 & 58,1 & 1.112 & 43,9 & 4.549 & 83,0 & 6.566 & 80,3 & 7.917 & 88,7 & 8.493 & 84,6 \\
\hline Santa Cruz & 116 & 0,0 & 1.330 & 80,0 & 3.809 & 88,7 & 5.380 & 85,3 & 4.510 & 88,4 & 5.669 & 86,6 \\
\hline Santa $\mathrm{Fe}$ & 10.060 & 50,1 & 15.267 & 55,8 & 33.763 & 66,8 & 37.774 & 66,1 & 57.538 & 75,2 & 52.395 & 70,3 \\
\hline $\begin{array}{l}\text { Santiago } \\
\text { del Estero }\end{array}$ & 1.733 & 69,8 & 4.697 & 80,2 & 9.976 & 87,3 & 13.346 & 87,6 & 15.556 & 90,0 & 17.730 & 89,1 \\
\hline $\begin{array}{c}\text { Tierra del } \\
\text { Fuego }\end{array}$ & 646 & 68,0 & 1.130 & 53,8 & 2.093 & 83,6 & 2.559 & 79,1 & 2.553 & 84,4 & 2.560 & 79,6 \\
\hline Tucumán & 681 & 50,2 & 1.883 & 60,1 & 4.549 & 66,7 & 10.766 & 66,6 & 30.531 & 83,6 & 29.313 & 81,8 \\
\hline
\end{tabular}

Fuente: Giacometti (2010) en base a datos de DINIECE. Relevamientos anuales.

Estos datos muestran que el incremento de la matrícula del nivel inicial ha sido sostenido, y se explica particularmente por la ampliación de la cobertura de 4 años y en menor medida la extensión de la oferta de sala de 3, aunque con claras heterogeneidades regionales: en Capital Federal y Provincia de Buenos Aires más de una cuarta parte de los alumnos corresponden a la sala de 3 años, en otras, como Mendoza o Misiones, no supera el 3\% lo cual da cuenta de que estos niños permanecen en el hogar al cuidado de la madre o de algún familiar directo.

A su vez, y tal como el Gráfico 4 muestra, la presencia de niños y niñas supone una disminución de la tasa de actividad femenina, pero la misma es mucho más intensa entre las mujeres que habitan en hogares con menos ingresos. 
Gráfico 4

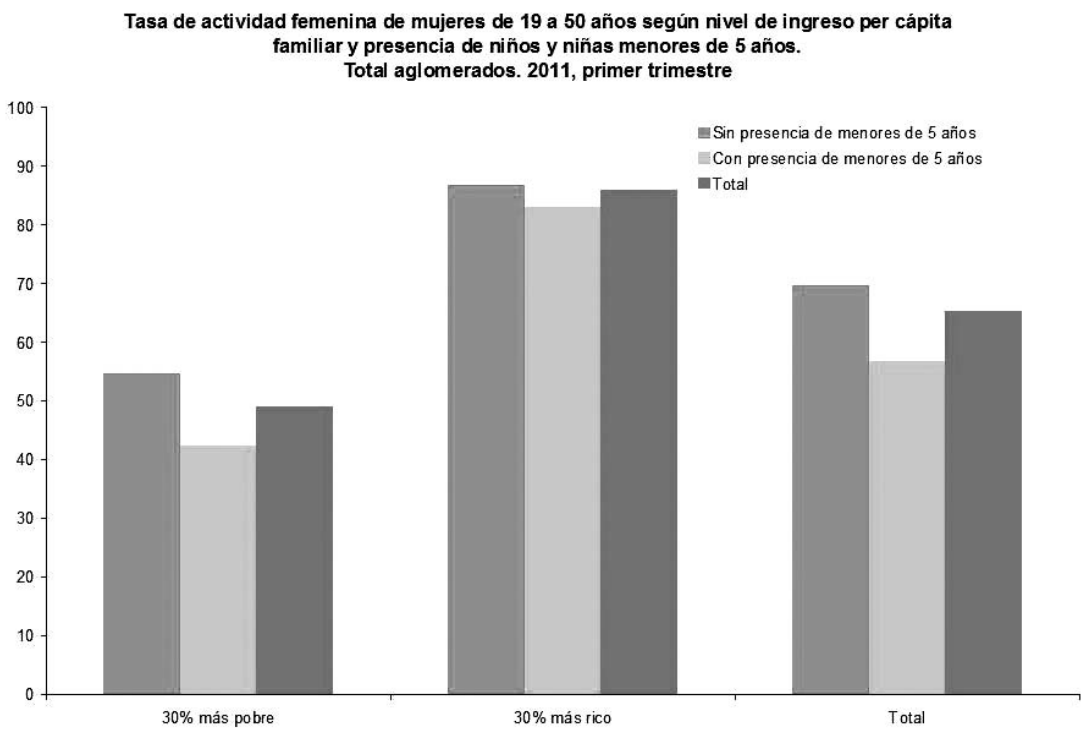

Fuente: Elaboración propia de Pautassi et al (2011) a partir de datos de EPH. INDEC.

Nuevamente, los datos expuestos permiten una primera aproximación parcial a la situación de cuidado de niños, niñas y adolescentes, sin que se acabe el universo de demandas de cuidado y donde la disponibilidad de información es aún más escasa cuando las demandas provienen de personas adultas mayores, enfermas o con discapacidades.

\section{¿Círculos concéntricos? La hora de la política social}

La confluencia de las necesidades de cuidado, la situación en que las mujeres se insertan laboralmente, las fronteras que su condición de contratación las incluye o excluye de las escasas regulaciones laborales establecidas al efecto, dan cuenta de la complejidad de la situación actual pero especialmente la urgencia que demanda su in- 
tervención. En la medida que no se considere, desde el Estado, las políticas sociales pero también los actores económicos, sociales, y la sociedad en su conjunto, que está en juego nada más ni nada menos que la reproducción social se seguirá responsabilizando a las mujeres que garanticen el cuidado de toda la sociedad. Carga por demás pesada pero absolutamente desigual, estratificada, injusta y violatoria del principio de igualdad de oportunidades.

¿Cómo salir del círculo? En primer lugar, el imperativo es que de una vez por todas se considere al cuidado como un derecho, individual, universal e indisponible, fundamentado en la potestad que tiene cada personas a ser cuidada, a cuidarse y a cuidar a los otros. En tanto derecho, implica la imposición de obligaciones positivas y negativas, tanto al Estado como a otros miembros de la sociedad, en el entendido que el cuidado es una responsabilidad social y no solo individual; y por lo tanto debe ser incorporada dentro del sistema de política sociales, independientemente de la inserción asalariada de la persona y no solo para las mujeres o de la cantidad de hijos e hijas.

Concordantemente, el enfoque de derechos demanda profundas reformas en el marco de las políticas públicas vigentes, ya que garantizar el cuidado como derecho universal es un proceso transversal, que implica producir información estadística confiable y no seguir ignorando $-\mathrm{y}$ por lo tanto excluyendo- los comportamientos vinculados. Del mismo modo, se deben incorporar indicadores con enfoque de género indispensables para el análisis del mercado de trabajo como herramienta para el diseño de políticas laborales integrales, de calidad y que consideren apuntar a garantizar trayectorias laborales equitativas. La producción de información debidamente desagregada a efectos de determinar cómo y de qué manera se resuelve la problemática del cuidado y su vinculación con la condición laboral de quien se encuentra cuidando, no corresponde únicamente con un medio para garantizar la efectividad de una política pública, sino constituye una obligación estatal.

Del mismo modo, es necesario promover una revisión de las políticas inconexas y discriminatorias vigentes, evaluando si las mismas son compatibles con una efectiva inclusión del cuidado como derecho 
universal y no particular para las mujeres, pero también adecuando todo el sistema a las obligaciones implícitas en este derecho, en particular en relación con el mercado de trabajo y el sistema de seguridad social, del mismo modo que el conjunto de las políticas sociales.

Concordantemente, se debe revisar el hecho que las escasas medidas de conciliación del trabajo con las responsabilidades familiares, herencia de los arreglos institucionales locales, el título de derecho está asociado a la condición de trabajadora asalariada formal, significando la condición maternal como excluyente para el acceso al cuidado de hijos y excluyendo a las trabajadoras informales y a aquellas trabajadoras domésticas, con o sin remuneración. Esto es, se ejerce el derecho a disponer de tiempo para cuidar (licencias maternales) y servicios para el cuidado (infraestructura) y recursos (asignaciones) en función de los derechos laborales acordados. Precisamente, estas medidas no deben ser sólo para los asalariados sino para cada persona, en cualquier momento del ciclo vital que se encuentre y que pueda cuidar y cuidarse.

Al mismo tiempo, se requiere que el Estado promueva con mayor fuerza la regularización del empleo, en términos de lograr que los Estados garanticen puestos de trabajo formales, es decir, registrados y que gocen de la protección del sistema de seguridad social, y respetuosos de la equidad de género. El comportamiento del mercado de trabajo en la Argentina de la post convertibilidad es un claro ejemplo de ello, ya que el crecimiento económico por sí mismo no implica la superación de las discriminaciones y desigualdades persistentes.

Del mismo modo, se deben revisar en forma urgente los marcos legales de regulación del trabajo doméstico remunerado (servicio doméstico) de modo de reformar todas las normas discriminadoras y poner a las trabajadoras ocupadas en este sector en igualdad de condiciones que los demás trabajadores asalariados. Es hora que el proyecto de ley de servicio doméstico, que equipara en las mismas condiciones que los demás trabajadores asalariados, con más de tres años en debate parlamentario se convierta en ley. Y como el mero hecho que se reconozca el derecho al cuidado no garantiza que se provea del mismo, en condiciones de igualdad, calidad y cantidad suficiente, 
es necesario que se diseñen políticas al tal efecto. Por lo mismo, no se puede salir de esta situación de discriminación y de exclusión de vastos sectores de la población de acciones de cuidado, con la misma oferta. Esto es, se deben complementar y transversalizar las acciones que abarcan el cuidado en toda su dimensión, proveyendo de infraestructura y servicios adecuados para varones y mujeres, ampliando las licencias para varones y parentales y revisando las actualmente existentes para mujeres y no solo vinculadas con el primer momento del ciclo vital de quienes deber ser cuidados; superar la línea divisoria de prestaciones vinculadas para asalariados formales de los informales, exigiendo a los empleadores privados el cumplimiento de su obligación de garantizar los medios y medidas de conciliación trabajofamilia; considerar la división sexual del trabajo y como promover un uso del tiempo más equitativo, disponer de medidas que promuevan el ejercicio de derechos de quienes demandan ser cuidados, revisar las lógicas contributivas y no contributivas del sistema de seguridad social de modo de no excluir de la posibilidad de cobertura a las mujeres que desempeñan tareas de cuidado de manera exclusiva.

Estas propuestas lejos están de ser exhaustivas y no agotan la discusión en torno a la inserción y trayectoria laboral de las mujeres como tampoco el reconocimiento del derecho al cuidado de cada persona, sino que buscan destacar la necesidad y urgencia de promover medidas efectivas superadoras de los círculos concéntricos y altamente inequitativos en los que se encuentran las mujeres, buscando alcanzar la equidad en el marco de la promoción de autonomía y de la igualdad que reconozca las diferencias. Es hora que la política social recupere su rol rector en la distribución del bienestar. 


\section{Referencias bibliográficas}

ABRAMO, L. y TODARO, R. Cuestionando un mito: costos laborales de hombres y mujeres en América Latina. Lima, Oficina Internacional del Trabajo, 2002.

BATTHYÁNY, K. Cuidado infantil y trabajo: ¿un desafío exclusivamente femenino? Montevideo, Centro Interamericano para el Desarrollo del Conocimiento en la Formación Profesional (CINTERFOR)/Oficina Internacional del Trabajo (OIT), 2004.

CEPAL. Panorama social de América Latina. Santiago de Chile, CEPAL, 2009.

EQUIPO LATINOAMERICANO DE JUSTICIA Y GÉNERO (ELA). De eso no se habla: el cuidado en la agenda pública: Estudio de opinión sobre la organización del cuidado. Buenos Aires, 2012.

FOLBRE, N. The invisible Heart. Economics and Family Values. New York, The New York Press, 2001.

GIACOMETTI, C. Diagnóstico de la situación educativa de las mujeres en América Latina y el Caribe hispano "Más allá de las metas del milenio". El Caso Argentino. Buenos Aires, CLADEM, 2010 (mimeo).

MARCO NAVARRO, F. y RICO, M. N. "Cuidado y políticas públicas: debates y estado de situación a nivel regional”, en: PAUTASSI, L. y ZIBECCHI, C. (comp.) Redefiniendo las fronteras del cuidado. Agenda, derechos e infraestructura. Buenos Aires, en edición, 2013.

MINISTERIO DE TRABAJO. El trabajo femenino en la postconvertibilidad. Argentina 2003-2007. Comisión Económica para América Latina y el Caribe, Colección Documentos de proyectos. Buenos Aires, 2009.

MINISTERIO DE TRABAJO. Boletín de Género. Buenos Aires, Ministerio de Trabajo, Empleo y Seguridad Social, 2010.

PAUTASSI, L. El cuidado como cuestión social desde un enfoque de derechos. Serie Mujer y Desarrollo N²7. Santiago de Chile, CEPAL, octubre 2007.

PAUTASSI, L., GIACOMETTI, C. y GHERARDI, N. Sistema previsional en Argentina y equidad de género. Situación actual (2003-2010) y perspectivas futuras. Serie Documentos de Trabajo Equipo Latinoamericano de Justicia y Género, Buenos Aires, Diciembre 2011. www.ela.org.ar

PAUTASSI, L. y RICO, M. N. "Licencias para el cuidado infantil. Derecho de hijos, padres y madres", en: Desafíos. Boletín de la infancia y adolescencia sobre el avance de Objetivos de desarrollo del Milenio. Santiago de Chile, CEPAL-UNICEF; N $^{\circ} 12$, julio de 2011, págs. 4-9.

PAUTASSI, L., FAUR E. y GHERARDI, N. Legislación laboral en seis países latinoamericanos. Avances y omisiones para una mayor equidad. Serie Mujer y Desarrollo N ${ }^{\circ}$ 56. Santiago de Chile, CEPAL, 2004. 
PNUD-OIT. Trabajo y familia: hacia nuevas formas de conciliación con corresponsabilidad social. Santiago de Chile, Programa de Naciones Unidas para el Desarrollo y Organización Internacional del Trabajo, 2009.

RICO, M. N. "Apuntes sobre la economía del cuidado". Unidad Mujer y Desarrollo, CEPAL. Documento interno, 2005.

RODRÍGUEZ ENRÍQUEZ, C. "La organización del cuidado de niños y niñas en la Argentina y el Uruguay", en: MONTAÑO VIRREIRA, S. y CALDERÓN MAGAÑA, C. (Coords). El cuidado en acción: entre el derecho y el trabajo. Santiago, Cuadernos de la Cepal 94, Julio 2010.

RODRÍGUEZ ENRÍQUEZ, C. Las politicas de conciliación empleo-responsabilidades de cuidado y la distribución del trabajo. Exploración de argumentos económicos. Ponencia presentada en el $9^{\circ}$ Congreso Nacional de Estudios del Trabajo, Asociación Argentina de Estudios del Trabajo (ASET). Buenos Aires, 5-7 Agosto 2009.

TORNS MARTIN, T. "De la imposible conciliación a los permanentes malos arreglos”, en: Cuadernos de Relaciones Laborales. Barcelona, España, No 23, 2005. pp. 15-33.

TRONTO, J. "Vicious Circle of Privatized Caring", in: Socializing Care: Feminist Ethics and Public Issues. Edited by Maurice Hamington and Dorothy Miller, Lanham, Maryland, Rowman and Littlefield, 2006. 


\section{a. Libros:}

APELLIDO, Nombre (del/a autor/a del libro). Título del libro. Lugar, Editorial, año.

b. Artículos o Capítulos de Libros:

APELLIDO, Nombre (del/a autor/a del artículo o capítulo). “Título del artículo o capítulo", en: APELLIDO, Nombre (del/a autor/a, compilador/a, organizador/a o editor/a del libro). Título del libro o revista. Lugar, Editorial, año. Páginas (del artículo o capítulo).

\section{c. Artículos de Publicación Periódica:}

APELLIDO, Nombre (del/a autor/a del artículo o capítulo). “Título del artículo o capítulo", en: Título de la Publicación. Año, Volumen, Número, $\mathrm{N}^{\circ}$ 37. Páginas (del artículo).

\section{d. Fuentes y/o documentos electrónicos:}

APELLIDO, Nombre (del/a autor/a del libro, artículo, ponencia, etc.). Título. Tipo de soporte (CD-ROM; página web; blog). Fecha de publicación. ISBN o ISSN [en línea: fecha de consulta]. Disponible en: link completo.

\section{Sistema de Arbitraje}

Para la aprobación de su publicación, los trabajos serán sometidos a la consideración del Consejo de Redacción y de evaluadores/as externos/as. Se tendrá en cuenta para su aprobación la pertinencia del tema propuesto, la calidad de la publicación, la actualización de la bibliografía y el cumplimiento de normas formales de redacción. En primer lugar, los artículos serán objeto de una evaluación preliminar por parte del Consejo de Redacción, quien determinará la pertinencia para su publicación. Una vez establecido que el artículo cumple tanto con los requisitos temáticos como formales, será enviado a dos pares académicos externos, quienes determinarán, de manera anónima: a) recomendar la publicación sin modificaciones; b) publicar con sugerencias de re-elaboración; c) no recomendar la publicación. Los resultados del proceso de evaluación serán inapelables en todos los 
casos. En caso de ser publicado el artículo, el/los autor/es transfieren todos los derechos de autor a Cátedra Paralela, sin cuyo permiso expreso no podrá reproducirse ninguno de los materiales allí publicados. Asimismo, Cátedra Paralela asume los derechos para editar, publicar, reproducir, distribuir copias, preparar trabajos derivados en papel y/o electrónicos e incluir el artículo en índices nacionales e internacionales o bases de datos. Se solicita a los/as colaboradores/as la entrega de la nota expresa de originalidad y cesión de los derechos de autor/a. La Revista Cátedra Paralela no se hace responsable por los trabajos no publicados ni se obliga a mantener correspondencia con los/as autores/as.

\section{Modelo Nota de Autorización - Revista Cátedra Paralela}

Por la presente autorizo a la Revista Cátedra Paralela, a la publicación del artículo de mi autoría

.........en el $\mathrm{N}^{\circ}$....... del año ......, publicación de la Escuela de Trabajo Social de la UNR y el Colegio de profesionales de Trabajo Social de la $2^{a}$ Circunscripción de la Provincia de Santa Fe.

Se deja constancia de que no corresponde retribución pecuniaria derivada del derecho de autor.

LUGAR Y FECHA:

FIRMA Y ACLARACIÓN: 